\title{
FAVORABILIDAD ECOGEOGRÁFICA PARA EL CORZO: DISTRIBUCIÓN Y ABUNDANCIA
}

\author{
P. ACEVEDO ${ }^{1,2^{*}}$, R. $\operatorname{REAL}^{1} \&$ C. GORTÁZAR ${ }^{2}$ \\ 1 Departamento de Biología Animal, Facultad de Ciencias, Universidad de Málaga, 29071 Málaga \\ *pacevedo@irec.csic.es; pacevedo@uma.es \\ 2 Instituto de Investigación en Recursos Cinegéticos (CSIC-UCLM-JCCM), 13071 Ciudad Real
}

\begin{abstract}
Species distribution models (SDM) are widely used in ecology, biogeography and conservation. Here, we modelled the distribution of roe deer (Capreolus capreolus) in mainland Spain in order to i) determine its macro-ecological requirements, ii) assess the favourability for this species and iii) explore the model usefulness as an index of the species relative abundance. Our results showed that roe deer distribution can be explained by predictors related with topography, climate, lithology, geography and human activity. Climate was the more relevant factor, followed by human activity, topography, spatial location and lithology. The model accurately predicted the species probability of occurrence and also, but weak$l y$, the species' relative abundance.
\end{abstract}

Keywords: Abundance, roe deer, distribution, favourability function, Capreolus capreolus.

RESUMEN.- Los modelos de distribución de especies (SDM) están siendo cada vez más usados para abordar cuestiones relacionadas con la ecología, biogeografía y conservación de las especies. En este estudio nos proponemos realizar un modelo de distribución para el corzo (Capreolus capreolus) en la España peninsular para estudiar los determinantes macroambientales de su rango de distribución, evaluar la favorabilidad para la especie, y la utilidad del modelo como indicador de su abundancia relativa. El modelo indica que su distribución se puede explicar en términos topográficos, climáticos, litológicos, geográficos y de presión humana. El clima fue el factor que en su efecto puro explicó un mayor porcentaje de variación, y le siguen la presión humana, la topografía, la localización espacial y la litología. El modelo obtenido predijo con elevada precisión la probabilidad de presencia de la especie y con cierto detalle su abundancia relativa.

Palabras clave: Abundancia, corzo, distribución, función de favorabilidad, Capreolus capreolus. 


\section{Introducción}

Las relaciones entre los gradientes ambientales y la supervivencia de las poblaciones de una especie pueden ser usadas para modelizar la respuesta potencial de la especie a tales gradientes (Austin et al., 1990). De este modo se pueden establecer mapas predictivos de potencialidad ambiental para la especie, así como describir las características macroambientales que las definen (Acevedo et al., 2007a). Esto es lo que algunos autores denominan distribución potencial -o incluso nicho ecológico- de la especie (Soberón \& Peterson, 2005), y a este tipo de herramientas analíticas se las conoce como modelos de distribución de especies o modelos de distribución de hábitat (Guisan \& Zimmermann, 2000).

Los modelos de distribución de especies (en adelante SDM) están siendo cada vez más usados para abordar cuestiones relacionadas con la ecología, biogeografía y conservación de las especies. Sus aplicaciones son muy variadas y -refiriéndonos por ejemplo a estudios realizados sobre ungulados ibéricos- abarcan desde el estudio de los requerimientos macroambientales de una especie o de un grupo de especies (Acevedo et al., 2007a), la evaluación de la potencialidad del territorio para especies invasoras (Cassinello et al., 2006), o el estudio de las relaciones biogeográficas entre diferentes taxones (Acevedo et al., 2007b; Delibes-Mateos et al., 2009; Acevedo \& Real, 2011), hasta la evaluación del efecto del cambio en el clima (Real et al., 2010) o de los usos del suelo (Acevedo et al., 2011) sobre su área de distribución.

La información necesaria para realizar un SDM generalmente proviene de atlas de distribución (Palomo et al., 2007), sobre todo cuando la escala espacial del análisis es regional o nacional, y de las coberturas digitales de información ambiental, disponibles muchas de ellas de manera gratuita en servidores de Internet (http:/ / edit.csic.es / GISdownloads.html). Desde esta información, la modelización permite obtener una medida continua del grado de adecuación del área de estudio para la especie. Pero la interpretación de lo que realmente representa dicha adecuación no puede realizarse de manera directa ya que precisa de ciertas consideraciones relacionadas tanto con el procedimiento de modelización (Jiménez-Valverde et al., 2008) como con la ecología de la especie que se está estudiando (Múñoz \& Real, 2006).

Algunos de los SDM implementados en programas amigables, como por ejemplo ENFA (Hirzel et al., 2002) o Maxent (Phillips et al., 2006), producen valores de adecuación de difícil interpretación debido a que en estos procedimientos se realiza un reescalamiento -se asigna a la localidad de máxima probabilidad el valor máximo que puede tomar el índice- de los valores de adecuación. Por ello, estos valores de adecuación sólo pueden interpretarse como valores relativos cuya utilidad consiste en el establecimiento de com- 
paraciones entre localidades (Acevedo et al., 2007a). Para poder ser interpretados en términos absolutos -en relación a alguna característica biológica de la especie-, es preciso realizar una validación previa de los valores predichos por el modelo con datos independientes. Esta validación consistirá en el análisis de la relación existente entre los valores de adecuación y los de un parámetro de la población (probabilidad de presencia, abundancia poblacional, calidad de la dieta,...) que estamos interesados en predecir. Sin embargo, los valores predichos mediante regresiones logísticas o modelos generalizados aditivos tienen en si mismos una interpretación directa ya que indican la probabilidad de presencia de la especie en cada una de las localidades (Acevedo \& Cassinello, 2009). En estos casos, se debe evaluar la calibración (Pearce \& Ferrier, 2000), es decir, testar el grado en el que los valores observados (prevalencia de la especie) se ajustan a los valores predichos por el modelo, por ejemplo ver si la especie está presente en el $40 \%$ de las localidades en las que el modelo predice una probabilidad de 0.4 .

Una interpretación similar -aunque no idéntica- a la probabilidad de presencia puede obtenerse usando la función de favorabilidad (Real et al., 2006), en la que los valores predichos son indicativos del grado en el que la probabilidad de presencia de una especie en una determinada localidad difiere de la probabilidad de encontrarla por azar en el área de estudio (prevalencia de la especie). Así, valores de favorabilidad superiores a 0.5 indican una probabilidad de presencia superior a la prevalencia (localidades con características ambientales que favorecen la presencia de la especie), y por debajo de dicho valor muestran una probabilidad inferior a la prevalencia (localidades con características ambientales desfavorables).

Pero los SDM no se quedan aquí, ya que en ocasiones se da un paso más y se evalúa su capacidad para predecir otros parámetros de la dinámica de la especie como por ejemplo la abundancia relativa de las poblaciones (Nielsen et al., 2005). Para ello podría ser usado cualquiera de los modelos anteriormente mencionados, ya que en este caso el interés no radica en el valor absoluto de las predicciones, tal y como ocurría con la probabilidad y la favorabilidad, sino en su valor relativo respecto al resto del área de estudio. En este punto, trabajos recientes han demostrado la utilidad de los modelos de hábitat para predecir la abundancia local de plantas y animales (Boyce et al., 2001; Pearce \& Ferrier 2001; Real et al., 2009). Sin embargo, los resultados mostrados en otros estudios muestran que la aplicabilidad de este tipo de modelos depende de numerosos factores, muchos de ellos intrínsecos de la especie a modelizar, por lo que a la hora de afrontar un modelo de abundancia el diseño del modelo debe contemplar, en mayor medida que los modelos dirigidos a determinar la distribución, aspectos de la biología de la especie (Pearce \& Ferrier, 2001; Nielsen et al., 2005), y las predicciones deben ser validadas con 
datos de abundancia, preferiblemente provenientes de muestreos independientes.

En este contexto nos proponemos realizar un modelo de distribución para el corzo (Capreolus capreolus) en la España peninsular con un triple objetivo: i) conocer los determinantes macroambientales de su actual rango de distribución, ii) evaluar la favorabilidad ambiental del territorio para la especie, y iii) determinar la utilidad del modelo como indicador de la abundancia relativa de la especie para así poder interpretar correctamente los patrones obtenidos desde el punto de vista de la gestión global de la especie.

\section{Métodos}

\subsection{Datos de distribución y caracterización ambiental}

El área de estudio sobre la que se ha realizado este análisis es la España peninsular, y las cuadrículas UTM de $100 \mathrm{~km}^{2}$ han sido las unidades territoriales consideradas $(n=5273)$. Los datos sobre la distribución de la especie han sido tomados del Atlas y Libro Rojo de los Mamíferos Terrestres de España (Palomo et al., 2007), y en Cuenca y Guadalajara esta información se ha actualizado con datos provenientes de muestreos de campo (Acevedo et al., 2010a; ver Figura 1).

Para la modelación de la distribución de corzo se han reunido 24 variables relacionadas con la localización espacial, la topografía, el clima, la litología y la actividad humana (Tabla 1). Las variables se eligieron en función de su disponibilidad a esta escala y de su potencial poder predictivo (ver Acevedo \& Real, 2011), asumiéndose que están al menos correlacionadas con factores causales de la distribución de las especies. Como Robertson et al. (2003) han sugerido, los modelos que dependen de relaciones indirectas entre variables ambientales y la presencia de las especies pueden predecir las distribuciones incluso mejor que los modelos mecanísticos -que utilizan variables más directamente relacionadas con la fisiología de las especies-, razón por la que, en este estudio se han considerado variables ambientales. La explicación sobre la metodología seguida para la obtención de las variables puede encontrarse en Barbosa et al. (2003).

\subsection{El modelo estadístico}

La modelización se ha realizado mediante regresión logística (Hosmer \& Lemeshow, 1989), y se ha aplicado la función propuesta por Real et al. (2006) 


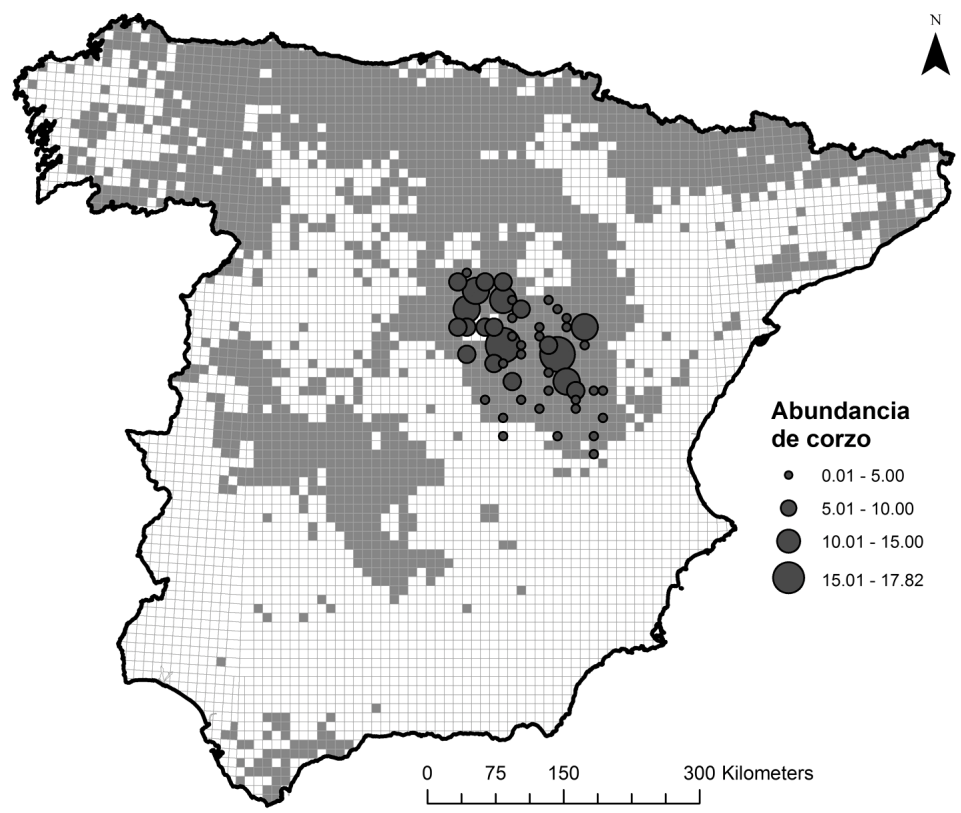

Figura 1. Las cuadrículas sombreadas en gris representan el área de distribución del corzo en la España peninsular (adaptado de Palomo et al., 2007). En Cuenca y Guadalajara estos datos fueron actualizados con los provenientes de Acevedo et al. (2010a). El tamaño de los círculos indica la abundancia relativa de las poblaciones de corzo consideradas en el estudio de Acevedo et al. (2010a) que serán las usadas para validar el modelo.

Figure 1. Roe deer distribution in mainland Spain (adapted from Palomo et al., 2007). These data were updated in Cuenca and Guadalajara according to Acevedo et al. (2010a). Circles size shows the roe deer relative abundance (Acevedo et al., 2010a) in those localities used to validate the model.

para obtener la favorabilidad del territorio para la especie. El modelo fue construido en dos pasos. En el primero de ellos se ha evaluado la relación de cada variable de manera independiente con la variable dependiente (presencia [ $n=2043]$ o ausencia [ $n=3230]$ de corzo en cada UTM de $100 \mathrm{~km}^{2}$ ). Tras este primer paso se ha considerado la tasa de descubrimientos falsos (FDR, de False Discovery Rate; Benjamini \& Hochberg, 1995), con el fin de minimizar la probabilidad de cometer un error de tipo I (ver otras aplicaciones por ejemplo en Acevedo \& Real 2011). Las variables seleccionadas tras el FDR han sido incluidas en el modelo final que se ha construido utilizando un procedimiento de selección por pasos hacia adelante-hacia atrás, con un umbral de significación de 0,05 para inclusión de una variable en el modelo y de 0,10 para su 
Tabla 1. Variables utilizadas para modelar la distribución de corzo en cuadrículas UTM de 10x10 $\mathrm{km}$ en la España peninsular.

Table 1. Variables used to model roe deer distribution in UTM $10 x 10 \mathrm{~km}$ squares in mainland Spain.

\begin{tabular}{|l|l|l|}
\hline Factor & Variable & Código \\
\hline Topografía & Altitud media (m) & Alti \\
& Pendiente media (grados) & Pend \\
\hline Clima & Número medio anual de días de helada & DiHe \\
& Número medio anual de días de granizo & DGra \\
& Número medio anual de días de niebla & DNie \\
& Variación anual de temperatura [=T Julio - T Enero $]$ & Vtem \\
& Insolación media anual (horas/ año) & Inso \\
& Radiación solar global media anual (Kwh/m²/ día) & RadS \\
& Evapotranspiración potencial media anual $(\mathrm{mm})$ & ETP \\
& Evapotranspiración real media anual (mm) $[=\mathrm{min}$. entre Prec y ETP] & ETR \\
& Precipitación media anual (mm) & Prec \\
& Precipitación máxima en 24 horas (mm) & PM24 \\
& Precipitación máxima relativa (mm) [=PM24 / PMed $]$ & PMR \\
& Número medio anual de días de precipitación $>0,1 \mathrm{~mm}$ & DiPr \\
& Irregularidad pluviométrica interanual & IrrP \\
& Índice de humedad relativa & IHum \\
& Humedad relativa media en enero a las 07:00 horas $(\%)$ & HuEn \\
& Humedad relativa media en julio a las 07:00 horas $(\%)$ & HuJu \\
\hline Litología & Permeabilidad del suelo & Perm \\
\hline Presión & Distancia mínima a una autopista o autovía (km) & DAut \\
humana & Distancia a un centro urbano de más de 100.000 habitantes $(\mathrm{km})$ & U100 \\
& Distancia a un centro urbano de más de 500.000 habitantes $(\mathrm{km})$ & U500 \\
\hline Situación & Latitud media (grados N) & Lati \\
espacial & Longitud media (grados E) & Long \\
\hline
\end{tabular}

eliminación. En una fase final de la modelización se ha evaluado la existencia de autocorrelación espacial sobre los residuos del modelo usando el estadístico I de Moran y siguiendo los procedimientos y razonamientos descritos en Diniz-Filho et al. (2003).

Los valores de probabilidad obtenidos con la regresión logística no dependen exclusivamente de las variables independientes, sino también de la probabilidad al azar debida a la proporción de presencias de la especie en el área de estudio (Hosmer \& Lemeshow, 1989). Cuando el número de presencias y el de ausencias en el área de estudio es diferente, los valores de probabilidad $(P)$ están sesgados hacia la categoría que presenta un mayor número de casos. Para obtener un valor predicho que fuera independiente de la prevalencia de la especie se ha descrito la función de favorabilidad (Real et al., 2006): 


$$
F=\frac{\frac{P}{(1-P)}}{\frac{n_{1}}{n_{0}}+\frac{P}{(1-P)}}
$$

Cuando el número de presencias $\left(n_{1}\right)$ es igual al número de ausencias $\left(n_{0}\right)$, $F=P$, pero si el número de presencias es menor que el de ausencias $F>P$, e inversamente, cuando el número de presencias es mayor que el de ausencias $F<P$. El umbral ambiental $(F=0,5)$ corresponde al valor esperado bajo igual favorabilidad en todas las localidades. Esto sería equivalente a asignar el valor $F=0.5$ a las condiciones ambientales por las cuales la $P$ es igual a la proporción de presencias de la especie en el área de estudio. Como anteriormente se ha comentado, los valores obtenidos utilizando la función de favorabilidad no reflejan probabilidad de presencia, sino favorabilidad del territorio, que es lo que normalmente se busca con la modelación de las distribuciones, principalmente en aquellas ocasiones en las que se precisa catalogar como favorable un área no ocupada por la especie o incluso como desfavorable una zona donde ésta sí ocurre. El modelo de favorabilidad es más adecuado que la probabilidad para dilucidar tendencias biogeográficas, para determinar el valor de conservación de distintos territorios, o para seleccionar las zonas más apropiadas para la reintroducción de especies.

El modelo desarrollado en este estudio tiene una componente predictiva y otra explicativa. El mejor modelo predictivo se obtiene considerando todos los predictores disponibles independientemente de la colinealidad existente entre ellos (Graham, 2003), tal y como hemos realizado en este estudio. Sin embargo, la colinealidad entre predictores afecta a la componente explicativa del modelo e imposibilita la descripción de relaciones simples entre cada predictor y la variable dependiente. En este caso, y para potenciar la capacidad explicativa del modelo, se ha realizado una partición de la variación con la que poder explorar la naturaleza funcional de las colinearidades (Borcard et al., 1992). Este procedimiento permite cuantificar la variación de una variable dependiente -en este caso los valores predichos por el modelo final- que es explicada por el efecto puro de cada factor, esto es, independientemente al resto de factores, así como la variación que se explica por el efecto combinado de dos o más factores. En este trabajo se ha seguido el procedimiento de sustracción descrito en Real et al. (2003), pero no se ha cuantificado la variación explicada por el efecto combinado entre dos o más factores debido a que el elevado número de factores considerado en este estudio (5; Tabla 1) dificulta mucho su interpretación (Acevedo \& Real, 2011).

Por otro lado, los valores predichos por el modelo han sido validados como indicadores de la probabilidad de presencia de la especie y también como indicadores de su abundancia relativa (Real et al., 2009). Lo primero se 
ha realizado mediante la evaluación del nivel de calibración del modelo para predecir la presencia de la especie (Pearce \& Ferrier, 2000). Para ello las localidades se han clasificado en 10 grupos de igual amplitud $(0,1)$ en función de la probabilidad predicha por el modelo en cada una de ellas. Sobre las localidades pertenecientes a cada una de estos grupos se ha calculado la prevalencia observada de la especie, medida como el porcentaje de las localidades pertenecientes al grupo en las que la especie está presente. Enfrentando la probabilidad predicha por el modelo con la prevalencia observada en cada uno de los grupos obtenemos el gráfico de calibración, en el que el ajuste perfecto (perfecta calibración) se obtiene cuando todos los puntos se sitúan en la línea de igualdad entre ambos ejes (diagonal). Hay descritos numerosos índices que permiten cuantificar el grado de ajuste a la diagonal, como por ejemplo el de Miller -mejores ajustes cuando toma valores más próximos a cero(Miller et al., 1991), que es el que ha sido usado en este estudio.

Para validar los valores predichos por el modelo como indicadores de la abundancia relativa de corzo se han usado datos provenientes de muestreos de campo, basados en el recuento de indicios de presencia de la especie, realizados en Cuenca y Guadalajara y que han sido publicados en Acevedo et al. (2010a). De este trabajo se pueden extraer datos para 61 localidades en las que la abundancia de corzo es altamente variable (rango 0,01 - 20,74 corzos / 100 ha [media \pm ET: $5,56 \pm 0,75]$ ). Dicho muestreo resulta muy adecuado ya que está basado en la misma unidad territorial que la usada en este estudio, es decir, las cuadrículas UTM de $100 \mathrm{~km}^{2}$. Por tanto la validación ha consistido en el análisis mediante una correlación de Spearman de la relación existente entre los valores de probabilidad/favorabilidad y la abundancia medida para las cuadrículas muestreadas en el trabajo anteriormente mencionado.

\section{Resultados}

Tras la exploración del FDR se excluyeron 3 variables (HuEn, PM24 y Vtem) del análisis multivariante. El modelo final se ha construido en 19 pasos que han incluido 17 variables en el modelo final para explicar la distribución de corzo en la España peninsular (Tabla 2). Los 5 factores considerados, esto es, situación espacial, topografía, clima, litología y actividad humana, han quedado representados en el modelo final. Los valores de probabilidad y de favorabilidad predichos para la especie se muestran en la Figura 2. En este caso, debido a que la prevalencia de la especie no difiere mucho de 0,5 los valores de probabilidad y favorabilidad son similares. Los resultados del estadístico I de Moran sólo evidenciaron la existencia de una autocorrelación espacial significativa a distancias cortas (menos de $20 \mathrm{~km}$ ) y con unos índices 
de Moran muy bajos (I media=-0,0002, rango -0,026 0,037). Por tanto, el modelo presentado en este trabajo, dada la reducida magnitud de las I de Moran y la reducida distancia a la que ha sido detectada la autocorrelación espacial en relación al tamaño de las unidades territoriales, la autocorrelación espacial no es considerado un problema (Diniz-Filho et al., 2003).

Tabla 2. Variables incluidas en el modelo final y sus parámetros estadísticos. Las abreviaturas de las variables como en la Tabla 1.

Table 2. Variables retained in the final model and their statistical parameters. Variables coded as in Table 1.

\begin{tabular}{|c|c|c|c|}
\hline Variable & Estimador & Wald & P-valor \\
\hline Pend & 0.159 & 74.533 & $<0.001$ \\
DGra & -0.107 & 26.595 & $<0.001$ \\
DiHe & 0.027 & 104.990 & $<0.001$ \\
DNie & $-1.75 \mathrm{E}-09$ & 5.608 & 0.018 \\
RadS & 0.016 & 55.922 & $<0.001$ \\
ETP & -0.003 & 7.022 & 0.008 \\
ETR & 0.004 & 98.894 & $<0.001$ \\
DiPr & -0.011 & 15.848 & $<0.001$ \\
IrrP & -0.234 & 141.724 & $<0.001$ \\
IHum & $5.87 \mathrm{E}-08$ & 14.886 & $<0.001$ \\
HuJu & 0.035 & 32.500 & $<0.001$ \\
Perm & -0.262 & 22.690 & $<0.001$ \\
DAut & -0.008 & 17.924 & $<0.001$ \\
U100 & 0.014 & 86.563 & $<0.001$ \\
U500 & 0.002 & 5.667 & 0.017 \\
Long & $-1.65 \mathrm{E}-06$ & 38.288 & $<0.001$ \\
Lati & $3.32 \mathrm{E}-06$ & 52.327 & $<0.001$ \\
Constante & -18.644 & 44.170 & $<0.001$ \\
\hline
\end{tabular}

La partición de la variación indica que de forma independiente al resto de factores, esto es su efecto puro, el factor climático es el que más variación explica del modelo final: $25,7 \%$. La contribución del resto de factores en su forma pura es más reducida, siendo 3,4\% para el factor presión humana, 2,7\% para el factor topográfico, el 2,6\% lo explica la situación espacial y el 0,8\% la litología. La reducida cantidad de variación explicada por el efecto puro de los factores considerados indica que la mayor parte de la variación se debe al efecto combinado de 2 o más factores.

El modelo obtenido para el corzo se muestra como perfectamente calibrado en lo que a probabilidad de presencia se refiere. El valor del índice de Miller es de 1E-06 y el gráfico de evaluación de la calibración se muestra en la Figura 3 y en él se aprecia un buen ajuste a lo largo de todo el rango de probabilidad predicha por el modelo. 


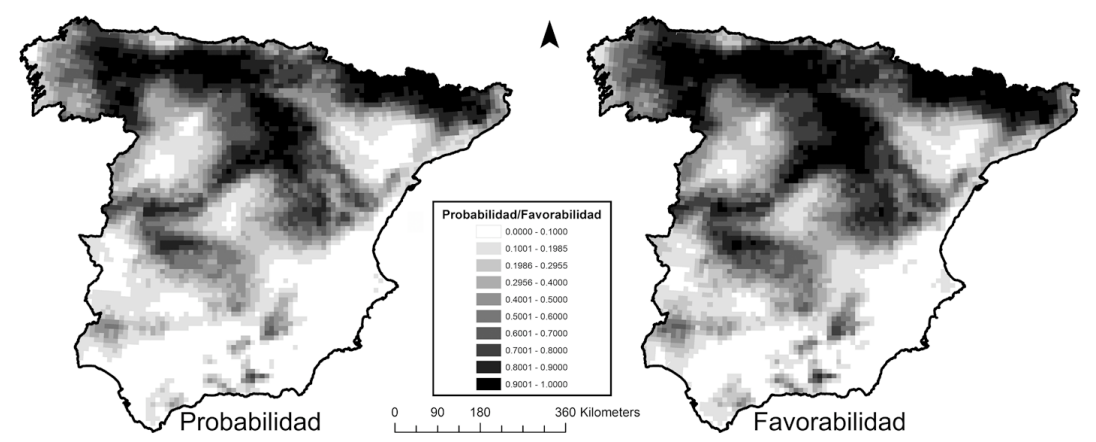

Figura 2. Valores de probabilidad y de favorabilidad para el corzo en la España peninsular de acuerdo al modelo presentado en la Tabla 2.

Figure 2. Probability of occurrence and favourability for roe deer in mainland Spain according to model shown in Table 2.

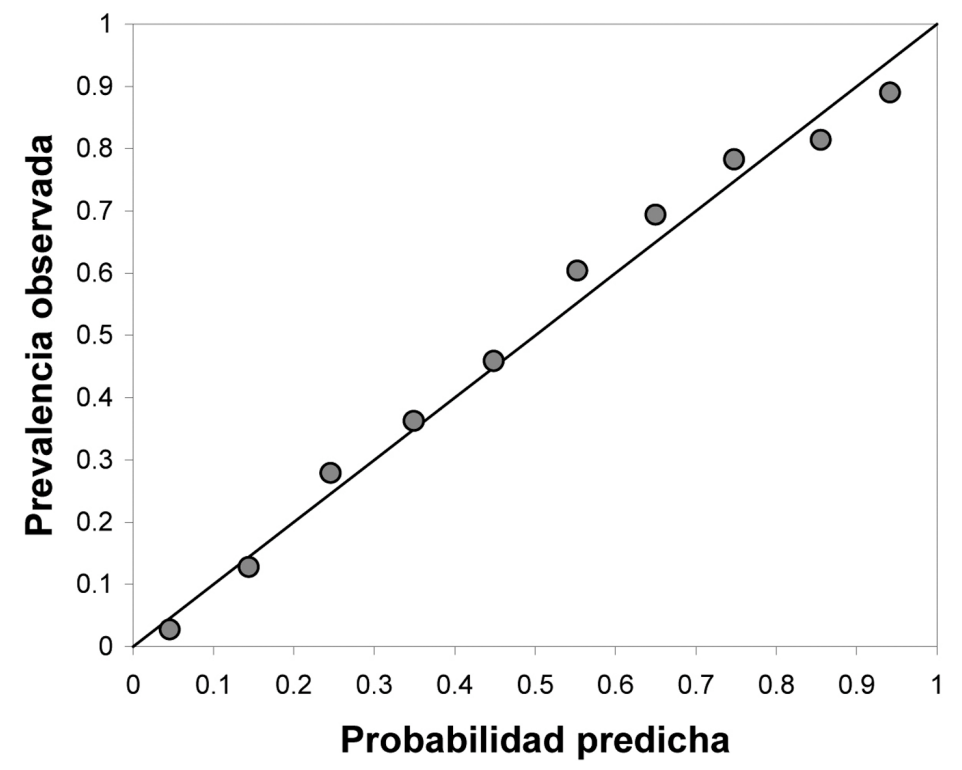

Figura 3. Gráfico de calibración en el que se muestra la relación entre la probabilidad de presencia predicha por el modelo (eje de abscisas) y la observada (eje de ordenadas). La línea diagonal indicaría la relación entre ambos valores cuando la calibración es perfecta, por lo que el error de los puntos respecto a esa línea es la base de los índices de calibración como el usado en este estudio.

Figure 3. Calibration plot showing the relationship between the predicted probability of occurrence and the observed proportion of evaluation sites occupied by the roe deer in mainland Spain. The graph is developed by plotting the proportion of evaluation sites found to be occupied within each of ten equi-interval predicted probability classes (perfect adjust on the diagonal). 
Respecto a la abundancia, los resultados indican que los valores predichos por el modelo se pueden consideran como un índice indirecto de la abundancia relativa de las poblaciones de corzo al estar correlacionadas significativamente con los valores de abundancia independientes $\left(\mathrm{r}_{\mathrm{s}}=0,323 ; \mathrm{p}=0,011\right.$; $\mathrm{n}=61$ ). Por tanto, los valores más altos de probabilidad/favorabilidad están señalando las localidades con mayor abundancia relativa de la especie.

\section{Discusión}

\subsection{Determinantes macroambientales y favorabilidad para el corzo}

El modelo obtenido indica que la distribución del corzo, y por tanto la favorabilidad ecogeográfica del territorio para esta especie, se puede explicar en términos topográficos, climáticos, litológicos, geográficos y de presión humana, aunque la importancia relativa del efecto puro de cada uno de ellos resultó ser bien distinta. Así el clima fue el factor que en su efecto puro explicó un mayor porcentaje de variación, y en orden de importancia le siguen la presión humana, la topografía, la situación espacial y la litología.

Es bien conocido el efecto del clima como determinante de la dinámica de las poblaciones de corzo, en particular, la precipitación diaria y la cobertura de nieve, y la temperatura y el viento han sido variables descritas previamente como condicionantes para esta especie (Aragón et al., 1995; Latham et al., 1997; Acevedo et al., 2005, 2010b). Estos trabajos evidencian el elevado poder explicativo que presentan las variables climáticas a la hora de estudiar los patrones de ocupación de las especies (Múñoz et al., 2005; Acevedo et al., 2010a). Los resultados aquí obtenidos indican que el agua útil es un requerimiento importante para el corzo ya que además de la utilidad directa para la especie, de manera indirecta se relaciona por ejemplo con la composición florística de las áreas ocupadas por corzo. La evapotranspiración real es la cantidad de agua que se evapora para la atmósfera, y es una medida de la disponibilidad simultánea de agua -relacionada a su vez con la permeabilidad del suelo y con la pendiente del terreno- y de energía biológicamente útiles (Márquez, 1996). Por su parte la irregularidad pluviométrica es el coeficiente de variación interanual de la precipitación y es una medida de la predecibilidad de las precipitaciones (Romero \& Real, 1996). La relación negativa detectada entre la irregularidad pluviométrica y la favorabilidad ecogeográfica para el corzo indica que las áreas por las que el corzo se distribuye presentan un régimen pluviométrico internanual más constante, es decir zonas en las que la influencia atlántica es más notoria. Análogamente, una relación nega- 
tiva entre la mediterraneidad y la presencia de corzo fue detectada estudiando la distribución del corzo en Francia (Gaudin et al., 1997).

El elevado peso relativo que presenta el factor climático explicando la distribución de esta especie sugiere una elevada sensibilidad de ésta a cambios en el clima (Real et al., 2010). Sería esperable, por tanto, que el corzo en la Península Ibérica viese alterada en una elevada magnitud su área de distribución en el futuro. Este resultado es coherente en el marco de la expansión que está experimentando la especie en la Península y que está modulada, en buena medida, por factores climáticos (Acevedo et al., 2005). Estudios realizados sobre otros ungulados ibéricos, por ejemplo la cabra montés Capra pyrenaica (Real et al., 2010), indican que su distribución se verá aumentada en un futuro próximo de acuerdo a los escenarios de cambio climático descritos por el Panel Intergubernamental de Cambio Climático (Nakicenovic et al., 2000). Además del clima, un aumento del área de distribución del corzo en un futuro reciente fue también predicho en relación a simulaciones de cambios en los usos del suelo (Acevedo et al., 2011).

De acuerdo con trabajos previos, la presión humana ha sido otro factor determinante de la distribución y abundancia del corzo (Hewison et al., 2001; pero ver Acevedo et al., 2005). La favorabilidad ecogeográfica de la Península para el corzo aumenta conforme lo hace la distancia a los núcleos urbanos. Los núcleos urbanos, además de ser una fuente de molestias para la fauna silvestre, son las áreas en las que más fuertemente se dejan notar las alteraciones del medio debido a actividades humanas. Por tanto son áreas poco adecuadas para esta especie que tradicionalmente ha estado vinculada a medios boscosos y escasamente alterados por el hombre (Aragón et al., 1995; Cargnelutti et al., 2002), si bien es cierto que la reciente expansión que está experimentando la especie hace que esta percepción del corzo como animal vinculado al bosque deba ser reconsiderada.

El hecho de que o bien variables geográficas puras o la combinación de las mismas en un polinomio (Legendre, 1993) queden retenidas en los modelos suele ser considerado como un reflejo indirecto de factores históricos que condicionaron fuertemente la distribución de la especie en el pasado (Real et al., 2008). En este caso concreto del corzo, esta especie se hallaba relegada en el siglo XIX en áreas muy concretas debido a un pastoreo intensivo, quemas y talas del monte, etc. Sin embargo dicha situación fue más crítica en la década de los 60 ya que, y presumiblemente debido a una elevada presión cinegética, se produjo una nueva retracción del área de distribución de la especie (Gortázar et al., 2000). Por ello, el corzo quedó restringido a determinados enclaves desde los que, y principalmente en las últimas décadas, comenzó un rápido proceso de recolonización (Acevedo et al., 2005) estando ahora ampliamente distribuido por la Península Ibérica, especialmente en su mitad norte 
(Figura 1). La distancia a estos núcleos de expansión ha sido descrita como el factor más relevante a la hora de explicar la expansión de la especie (Acevedo et al., 2005), estando esto en concordancia con los resultados del modelo predictivo aquí presentado.

En lo que a la interpretabilidad de los modelos se refiere, los resultados indican que, con elevada fiabilidad, los valores predichos por el modelo pueden ser considerados como indicadores de la probabilidad de presencia de la especie (Figura 3). Estos valores de probabilidad de presencia son de utilidad para completar datos de distribución provenientes de muestreos incompletos, y establecer una información de base en la que basar el diseño de posteriores muestreos (Guisan et al., 2006). En otras ocasiones el interés radica en conocer el grado en el que las condiciones ambientales de una determinada localidad potencian la presencia de la especie, y eso se obtiene aplicando la función de favorabilidad (Real et al., 2006). Este concepto de favorabilidad es muy relevante desde el punto de vista biogeográfico y su utilidad aumenta aun más cuando el interés del estudio consiste en realizar comparaciones entre especies/modelos (Acevedo et al., 2010b). La posibilidad de realizar comparaciones viene dada por la independencia que presenta la favorabilidad de la prevalencia de la especie lo que hace que los valores predichos de diferentes modelos sean directamente comparables, y que puedan ser usadas las reglas de la lógica difusa para poder relacionarlos (Real et al., 2009; Acevedo et al., 2010b, 2011). Conviene tener claros por tanto ambos conceptos (probabilidad y favorabilidad) con el fin de poder aplicar una función y otra -o las dos como en este caso- según las necesidades de la investigación. En este estudio, y debido a que la prevalencia de la especie es próxima a 0,5 (Real et al., 2006), el patrón de probabilidad y el de favorabilidad y así como sus valores son muy similares (Figura 2). Sin embargo, creímos necesario incluir ambas funciones en este manuscrito para clarificar ambos conceptos en el ámbito de los SDM.

\subsection{Abundancia relativa}

Los resultados indican que las predicciones del modelo realizado para el corzo pueden ser interpretadas como indicadores indirectos de la abundancia relativa de las poblaciones de corzo, al menos en las provincias de Cuenca y Guadalajara que es donde el modelo ha sido validado. Disponer de una estimación de la abundancia relativa de una especie para un territorio amplio es sumamente costoso en términos económicos y de esfuerzo por lo que, habitualmente, para ello se suele recurrir a la colaboración de voluntarios para realizar los muestreos de campo (Daniels, 2006). Por otro lado, trabajando con especies cinegéticas se pueden usar los datos de las capturas en las activida- 
des cinegéticas ya que éstos suponen una información de utilidad para tal fin (Mysterud et al., 2007), si bien el éxito de las actividades cinegéticas no depende exclusivamente de la abundancia de la especie (Acevedo et al., 2009) por lo que dicha información debe ser manejada con cautela sobretodo cuando se consideran territorios sometidos a diferentes regímenes de explotación cinegética. En este contexto, la determinación de un indicador de abundancia poblacional a lo largo del territorio que pueda ser obtenido desde datos de distribución de la especie -como el aquí evaluado- se muestra como una alternativa rentable y eficaz (Real et al., 2009) que, dada su relevancia, debería ser objeto de más profundas investigaciones.

La necesidad de disponer de información sobre la abundancia de una especie a lo largo del territorio se articula a muy diversos niveles. Desde el punto de vista de la fauna cinegética, resulta necesario en muchas ocasiones disponer de una estimación de la abundancia de la especie con el fin de poder adecuar los planes de gestión a las particularidades de cada localidad, pero sin perder de vista el contexto global que presenta la especie en todo su área de distribución (Farfán et al., 2008; Real et al., 2009). Por otro lado, la tasa de transmisión de patógenos en una población está íntimamente relacionada con su abundancia relativa (ver ejemplos en Gortázar et al., 2006). Así, la estimación de la abundancia de una especie en todo su área de distribución es un proceso clave para poder comprender el ciclo epidemiológico de los patógenos en los que dicha especie juega un papel relevante bien como vector $\mathrm{u}$ hospedador. Por ejemplo, la abundancia de ciervo (Cervus elaphus), estimada mediante SDM, ha sido recientemente utilizada para estudiar el papel que ésta juega en la abundancia del principal vector del virus de la lengua azul (Culicoides imicola) en la Península Ibérica (Acevedo et al., 2010c). Desde el punto de vista de la sanidad animal es necesario también conocer la abundancia de las poblaciones para poder establecer planes de contingencia frente a la entrada de un patógeno en el territorio como los que actualmente están emergiendo en la Península Ibérica (Ruiz-Fons et al., 2008).

\section{Agradecimientos}

Este trabajo ha sido promovido y financiado por la Consejería de Medio Ambiente de la Junta de Comunidades de Castilla - La Mancha. No podemos dejar de agradecer la ayuda proporcionada a todos los propietarios y gestores de fincas privadas que nos han facilitado el trabajo de campo realizado. P. Acevedo disfruta de un contrato dentro del programa Juan de la Cierva Fondo Social Europeo y sus investigaciones están parcialmente financiadas por el proyecto del Plan Nacional CGL2009-11316 - FEDER. 


\section{Referencias}

Acevedo, P. \& Cassinello, J., 2009. Human-induced range expansion of wild ungulates causes niche overlap between previously allopatric species: red deer and Iberian ibex in mountainous regions of southern Spain. Annales Zoologici Fennici, 46: 39-50.

Acevedo, P. \& Real, R., 2011. Biogeographical differences between the two Capra pyrenaica subspecies, C. p. victoriae and C. p. hispanica, inhabiting in the Iberian Peninsula: conservation implications. Ecological Modelling, 222: 814-823.

Acevedo, P., Delibes-Mateos, M., Escudero, M.A., Vicente, J., Marco, J. \& Gortázar, C., 2005. Environmental constraints in the colonization sequence of roe deer (Capreolus capreolus Linnaeus, 1758) across the Iberian Mountains, Spain. Journal of Biogeography, 32: 1671-1680.

Acevedo, P., Cassinello, J. \& Gortázar, C., 2007a. The Iberian ibex is under an expansion trend but displaced to suboptimal habitats by the presence of extensive goat livestock in central Spain. Biodiversity and Conservation, 16: 3361-3376.

Acevedo, P., Cassinello, J., Hortal, J. \& Gortázar, C., 2007b. Invasive exotic aoudad (Ammotragus lervia) as a major threat to native Iberian ibex (Capra pyrenaica): a habitat suitability model approach. Diversity and Distributions, 13: 587-597.

Acevedo, P., Vicente, J., Alzaga, V. \& Gortázar, C., 2009. Wild boar abundance and hunting effectiveness in Atlantic Spain: Environmental constraints. Galemys, 21: 13-29.

Acevedo, P., Ferreres, J., Jaroso, R., Durán, M., Escudero, M.A., Marco, J. \& Gortázar, C., 2010a. Estimating roe deer abundance from pellet group counts in Spain: An assessment of methods suitable for Mediterranean woodlands. Ecological Indicators, 10: 1226-1230.

Acevedo, P., Ward, A.I., Real, R. \& Smith, G.C., 2010b. Assessing biogeographical relationships of ecologically related species using favourability functions: a case study on British deer. Diversity and Distributions, 16: 515528.

Acevedo, P., Farfán, M.A., Márquez, A.L., Delibes-Mateos, M., Real, R. \& VargasS, J.M., 2011. Past, present and future of wild ungulates in relation to changes in land use. Landscape Ecology, DOI: 10.1007/ s10980-0109538-2.

Acevedo, P., Ruiz-Fons, F., Estrada, R., Márquez, A.L., Miranda, M.A., Gortázar, C. \& Lucientes, J., 2010c. A broad assessment of factors determining Culicoides imicola abundance: modelling the present and forecast- 
ing its future in climate change scenarios. PLOS ONE 5 (12): e14236. doi:10.1371/ journal.pone.0014236

Aragon, S., Braza, F. \& San José, C., 1995. Socioeconomic, physiognomic, and climate factors determining the distribution pattern of roe deer Capreolus capreolus in Spain. Acta Theriologica, 40: 37-43.

Austin, M.P., Nicholls, A.O. \& Margules, C.R., 1990. Measurement of the realized qualitative niche: environmental niches of five Eucalyptus species. Ecological Monographs, 60: 161-177.

Barbosa, A.M., Real, R., Olivero, J. \& Vargas, J.M., 2003. Otter (Lutra lutra) distribution modeling at two resolution scales suited to conservation planning in the Iberian Peninsula. Biological Conservation, 114: 377-387.

Benjamini, Y. \& Hochberg, Y., 1995. Controlling the false discovery rate: A practical and powerful approach to multiple testing. Journal of the Royal Statistical Society, Series B, 57: 289-300.

Borcard, D., Legendre, P. \& Drapeau, P., 1992. Partialling out the spatial component of ecological variation. Ecology, 73: 1045-1055.

Boyce, M.S., Mackenzie, D.I., Manly, B.F.J., Haroldson, M.A. \& Moody, D., 2001. Negative binomial models for abundance estimation of multiple closed populations. Journal of Wildlife Management, 65: 498-509.

Cargnelutti, B., Reby, D., Desneux, L. \& Angibault, J.M., 2002. Space use by roe deer in a fragmented landscape, some preliminary results. Revue Ecologie-Terre et Vie, 57: 29-37.

Cassinello, J., Acevedo, P. \& Hortal, J., 2006. Prospects for population expansion of the exotic aoudad (Ammotragus lervia; Bovidae) in the Iberian Peninsula: clues from habitat suitability modelling. Diversity and Distributions, 12: 666-678.

Daniels, M.J, 2006. Estimating red deer Cerous elaphus populations: an analysis of variation and cost-effectiveness of counting methods. Mammal Review, 36: 235-247.

Delibes-Mateos, M., Farfán, M.A., Olivero, J., Márquez, A.L. \& Vargas, J.M., 2009. Long-term changes in game species over a long period of transformation in the Iberian Mediterranean landscape. Environmental Management, 43: 1256-1268.

Diniz-Filho, J.A.F., Bini, L.M. \& Hawkins, B.A., 2003. Spatial autocorrelation and red herrings in geographical ecology. Global Ecology and Biogeography, 12: 53-64.

Farfán, M.A., Vargas, J.M., Guerrero, J.C., Barbosa, A.M., Duarte, J. \& Real, R., 2008. Distribution modelling of wild rabbit hunting yields in its original area (S Iberian Peninsula). Italian Journal of Zoology, 75: 161-172. 
Gaudin, J.C., Maillard, D. \& Gaillard, J.M., 1997. Colonisation par le chevreuil (Capreolus capreolus L.) de la zone méditerranéenne Française. Revue d'Ecologie - la Terre et la Vie, 52: 113-122.

Gortázar, C., Herrero, J., Villafuerte, R. \& Marco, J., 2000. Historical examination of the distribution of large mammals in Aragón, Northeastern Spain. Mammalia, 61: 411-422.

Gortázar, C., Acevedo, P., Ruiz-Fons, F. \& Vicente, J., 2006. Disease risks and overabundance of game species. European Journal of Wildlife Research, 52: 81-87.

Graham, M. H. (2003). Confronting multicollinearity in ecological multiple regression. Ecology, 84(11): 2809-2815.

Guisan, A. \& Zimmermann, N.E., 2000. Predictive habitat distribution models in ecology. Ecological Modelling, 135: 147-186.

Guisan, A., Broennimann, O., Engler, R., Vust, M., Yoccoz, N.G., Lehmann, A. \& Zimmermann, N.E., 2006. Using niche-based models to improve the sampling of rare species. Conservation Biology, 20: 501-511.

Hewison, A.J.M., Vincent, J.P., Joachim, J., Angibault, J.M., Cargnelutti, B. \& Cibien, C., 2001. The effects of woodland fragmentation and human activity on roe deer distribution in agricultural landscapes. Canadian Journal of Zoology - Revue Cannadiene de Zoologie, 79: 679-689.

Hirzel, A.H., Hausser, J., Chessel, D. \& Perrin, N., 2002. Ecological-niche factor analysis: How to compute habitat suitability maps without absence data? Ecology, 83: 2027-2036.

Hosmer, D.W. \& Lemeshow, S., 1989. Applied logistic regression. Wiley, New York.

Jiménez-Valverde, A., Lobo, J.M. \& Hortal, J., 2008. Not as good as they seem: the importance of concepts in species distribution modelling. Diversity and Distributions, 14:885-890.

Latham, J., Staines, B.W. \& Gorman, M.L., 1997. Correlations of red (Cervus elaphus) and roe (Capreolus capreolus) deer densities in Scottish forests with environmental variables. Journal of Zoology, 242: 681-704.

Legendre, P., 1993. Spatial autocorrelation: trouble or new paradigm? Ecology, 74: $1659-1673$

Márquez, A.L., 1996. Análisis biogeográfico de la pteridoflora ibérica. Tesis Doctoral, Universidad de Málaga.

Miller, M.E., Hui, S.L. \& Tierney, W.M., 1991. Validation techniques for logistic regression models. Statistics in Medicine, 10: 1213-1226.

Muñoz, A.R., Real, R., Barbosa, A.M., \& Vargas, J.M., 2005. Modelling the distribution of Bonelli's eagle in Spain: implications for conservation planning. Diversity and Distributions, 11: 477-486. 
Muñoz, A.R. \& Real, R., 2006. Assessing the potential range expansion of the exotic monk parakeet in Spain. Diversity and Distributions, 12: 656-665.

Mysterud, A., Meisingset, E.L., Veiberg, V., Langvatn, R., Solberg, E.J., Loe, E.L. \& Stenseth, N.C., 2007. Monitoring population size of red deer Cervus elaphus: an evaluation of two types of census data from Norway. Wildlife Biology, 13: 285-298.

Nakicenovic, N., Alcamo, J., Davis, G., De Vries, B., Fenhann, J., Gaffin, S., Gregory, K., Grübler, A., Jung, T.Y., Kram, T., La Rover, E.L., Michaelis, L., Mori, S., Morita, T., Pepper, W., Pitcher, H., Price, L., Raihi, K., Roehrl, A., Rogner, H.H., Sankovski, A., Schlesinger, M., Shukla, P., Smith, S., Swart, R., Van Rooijen, S., Victor, N. \& Dadi, Z., 2000. IPCC special report on emissions scenarios. Cambridge University Press, Cambridge.

Nielsen, S.E., Johnson, C.J., Heard, D.C. \& Boyce, M.S., 2005. Can models of presence-absence be used to scale abundance? Two cases studies considering extremes in life history. Ecography, 28: 1-12.

Palomo, L.J., Blanco, J.C. \& Gisbert, J., 2007. Atlas y libro rojo de los mamíferos terretres de España. Dirección General de Conservación de la NaturalezaSECEM-SECEMU, Madrid.

Pearce, J. \& Ferrier, S., 2000. Evaluating the predictive performance of habitat models developed using logistic regression. Ecological Modelling, 133: 225245.

Pearce, J. \& Ferrier, S., 2001. The practical value of modelling relative abundance of species for regional conservation planning: a case study. Biological Conservation, 98: 33-43.

Phillips, S.J., Anderson, R.P. \& Schapire, R.E., 2006. Maximum entropy modeling of species geographic distributions. Ecological Modelling, 190: 231259.

Real, R., Barbosa, A.M., Porras, D., Kin, M.S., Márquez, A.L., Guerrero, J.C., Palomo, L.J., Justo, E.R. \& Vargas, J.M., 2003. Relative importance of environment, human activity and spatial situation in determining the distribution of terrestrial mammal diversity in Argentina. Journal of Biogeography, 30: 939-947.

Real, R., Barbosa, A.M. \& Vargas, J.M., 2006. Obtaining environmental favourability functions from logistic regression. Enviromental and Ecological Statistics, 13: 237-245.

Real, R., Márquez, A.L., Estrada, A., Román Múñoz, A. \& Vargas, J.M., 2008. Modelling chorotypes of invasive vertebrates in mainland Spain. Diversity and Distributions, 14: 364-373.

Real, R., Barbosa, A.M., Rodríguez, A., García, F.J., Vargas, J.M., Palomo, L.J. \& Delibes, M., 2009. Conservation biogeography of ecologically interact- 
ing species: the case of the Iberian lynx and the European Rabbit. Diversity and Distributions, 5: 390-400.

Real, R., Márquez, A.L., Olivero, J. \& Estrada, A., 2010. Are species distribution models in climate warming scenarios useful for informing emission policy planning? An uncertainty assessment using fuzzy logic. Ecography, 33: 304-314.

Robertson, M.P., Peter, C.I., Villet, M.H. \& Ripley, B.S., 2003. Comparing models for predicting species' potential distributions: a case study using correlative and mechanistic predictive modelling techniques. Ecological Modelling, 164: 153-167

Romero, J. \& Real, R. 1996. Macroenvironmental factors as ultimate determinants of the distribution of common toad and netterjack toad in the south of Spain. Ecography, 19: 305-312

Ruiz-Fons, F., Reyes-García, A.R., Alcaide, V. \& Gortázar, C., 2008. Spatial and temporal evolution of bluetongue virus in wild ruminants, Spain. Emerging Infectious Diseases, 14: 951-953.

Soberón, J. \& Peterson, A.T., 2005. Interpretation of models of fundamental ecological niches and species' distribution areas. Biodiversity Informatics, 2: $1-10$. 